\title{
Editorial
}

\section{Space Dynamics}

\section{Antonio F. Bertachini A. Prado, ${ }^{1}$ Maria Cecilia Zanardii, ${ }^{2}$ Tadashi Yokoyama, ${ }^{3}$ and Silvia Maria Giuliatti Winter ${ }^{2}$}

${ }^{1}$ INPE-DMC, Brazil

${ }^{2}$ FEG-UNESP, Guaratinguetá, Brazil

${ }^{3}$ UNESP, Campus de Rio Claro, Brazil

Correspondence should be addressed to Antonio F. Bertachini A. Prado, prado@dem.inpe.br

Received 31 December 2009; Accepted 31 December 2009

Copyright (c) 2009 Antonio F. Bertachini A. Prado et al. This is an open access article distributed under the Creative Commons Attribution License, which permits unrestricted use, distribution, and reproduction in any medium, provided the original work is properly cited.

The space activity in the world is one of the most important achievements of mankind. It makes possible live communications, exploration of Earth resources, weather forecast, accurate positioning and several other tasks that are part of our lives today.

The space dynamics plays a very important rule in these developments, since its study allows us to plan how to launch and control a space vehicle in order to obtain the results we need.

This field considers the study of Celestial Mechanics and Control applied to spacecraft and natural objects. The main tasks are to determine the orbit and the attitude of the spacecraft based in some observations, to obtain its position and attitude in space in a given time from some initial conditions, to find the best way to change their orbits and attitude, to analyze how to use the information of the satellites to find the position and the velocity of a given point (e.g., a personal receptor, a satellite or a car), etc.

This field of study comes from Astronomy. The main contributors from the past have important names like Johannes Kepler (1571-1630) and Isaac Newton (1642-1727). Based on the observations of the motion of the planets realized by Tycho Brahe (1546-1601), Kepler formulated the three basic laws, which govern the motion of the planets around the Sun. From these laws, Newton formulated the universal Law of Gravitation. According to this Law, mass attracts mass in a ratio that is proportional to the product of the two masses involved and inversely proportional to the square of the distance between them. Those laws are the scientific bases of the space exploration age that officially begin with the launch of the satellite Sputnik in 1957 by the former Soviet Union. Since then, a strong battle between the United States of America (USA) and the Soviet Union took place leading to many achievements in Space. One of the most important results was the landing of the man on the Moon, achieved 
by the USA in 1969. From this point, several different applications of the space research were developed, changing for better the human life on Earth.

In that scope, this special issue of Mathematical Problems in Engineering is focused on the recent advances in space dynamics techniques. It has a total of 21 papers that are briefly described below.

Four of them are concerned with the attitude motion, control and determination. Optimal On-Off Attitude Control for the Brazilian Multimission Platform Satellite by G. Arantes Jr. et al. is the first one. This work deals with the analysis and design of the reaction thruster attitude control for the Brazilian Multi-Mission Platform satellite. The aim of this work is to provide smoother control for improved pointing requirements with less thruster activation or propellant consumption. The fuel is a deciding factor of the lifetime of the spacecraft and reduced propellant consumption is highly required, specially, regarding a multi-mission spacecraft wherein different payloads are being considering. The three-axis attitude control is considered and it is activated in pulse mode. Consequently a modulation of the torque command is compelling in order to avoid high non-linear control action. The paper considers the Pulse-Width Pulse-Frequency (PWPF) modulator, composed of a Schmidt trigger, a first order filter, and a feedback loop. This modulator holds several advantages over classical bang-bang controllers such as close to linear operations, high accuracy, and reduced propellant consumption. The Linear Gaussian Quadratic (LQG) technique is used to synthesize the control law during stabilization mode and the modulator is used to modulate the continuous control signal to discrete one. The results of the numerical simulations show that the obtained on-off thruster reaction attitude control system, based on the LQG/PWPF modulation, is optimal with respect to the minimization of the quadratic cost function of the states and control signals and propellant consumption. The paper presents a set of optimal parameter for the PWPF modulator by considering static and dynamics analysis. The obtained results demonstrate the feasibility of combining LQG/PWPF modulator in a unique controller for on-off thruster reaction attitude control system. Stability remains by adding the PWPF modulator and reasonable accuracy in attitude is achieved. Practical aspects are included in this study as filtering and presence of external impulsive perturbations. The advantages of less spent propellant shall contribute to the Brazilian Multi-Mission Platform project, specially, a satellite conceived to be used on a large number and different types of missions, in the context of an ever-advancing Brazilian space program.

The second paper on this subject is Highly Efficient Sigma Point Filter for Spacecraft Attitude and Rate Estimation by C. Fan and Z. You. In this paper, for spacecraft attitude determination problem, the multiplicative extended Kalman filter MEKF and other similar algorithms, have been good solutions for most nominal space missions. However, nowadays, due to their overload computational complexity, they are prohibitive for actual on board implementation. In this paper, the authors present a new and quite competitive algorithm, with significant lower computational complexity even when compared to the reduced sigma point algorithms. The precision is the same as the traditional unscented Kalman filters. In terms of efficiency, the proposed algorithm rivals MEKF, even in severe situations.

The next one is Spin-Stabilized Spacecraft: Analytical Attitude Propagation Using Magnetic Torques by R. V. Garcia et al.. This paper considers the problem in obtaining the attitude of a satellite in a given time based on information from a previous time. It analyzes the rotational motion of a spin stabilized Earth artificial satellite. It makes derivation of an analytical attitude prediction. Particular attention is given to torques, which come from residual magnetic and eddy currents perturbations, as well as their influences on the satellite 
angular velocity and space orientation. A spherical coordinated system, fixed in the satellite, is used to locate the spin axis of the satellite in relation to the terrestrial equatorial system.

The last paper of this topic is Using of H-Infinity Control Method in Attitude Control System of Rigid-Flexible Satellite by X. C. M. Cubillos and L. C. G. Souza. This paper considers the attitude control systems of satellites with rigid and flexible components. In the current space missions, this problem is demanding a better performance, which implies in the development of several methods to approach this problem. For this reason, the methods available today need more investigation in order to know their capability and limitations. Therefore, in this paper, the H-Infinity method is studied in terms of the performance of the Attitude Control System of a Rigid-Flexible Satellite.

There were four papers studying the problem of finding space trajectories. The first one is Hill Problem Analytical Theory to the Order Four: Application to the Computation of Frozen Orbits around Planetary Satellites by M. Lara and J. F. Palacián. In this paper, applications to the computation of frozen orbits around planetary satellites are made. The Hill problem, a simplified model of the restricted three-body problem, also gives a very good approximation for the dynamics involving the motion of natural and artificial satellites, moons, asteroids and comets. Frozen orbits in the Hill problem are determined through the double averaged problem. The developed method provides the explicit equations of the transformation connecting averaged and non averaged models, making the computation of the frozen orbits straightforward.

The second one covering this topic is Collision and Stable Regions around Bodies with Simple Geometric Shape by A. A. Silva et al.. Collision and stable regions around bodies with simple geometric shape are studied. The gravitational potential of two simple geometric shapes, square and triangular plates, were obtained in order to study the orbital motion of a particle around them. Collision and stable regions were also derived from the well known Poincaré surface of section. These results can be applied to a particle in orbit around an irregular body, such as an asteroid or a comet.

The next paper is Dynamical Aspects of an Equilateral Restricted Four-Body Problem by M. Álvarez-Ramírez and C. Vidal. It is an immediate extension of the classical restricted three body problem (ERFBP): a particle is under the attraction of three nonzero masses $\left(m_{1}, m_{2}, m_{3}\right)$ which move on circular orbits around their center of mass, fixed at the origin of the coordinate system in a such way that their configuration is always an equilateral triangle. In particular, it is assumed $m_{2}=m_{3}$. In a synodical system, a first integral of the problem is obtained. Using Hamiltonian formalism the authors define Hill's regions. Equilibrium solutions are obtained for different cases and the number of them depends on the values of the masses. The Lyapunov stability of these solutions is studied in the symmetrical case assuming $m_{1}=m_{2}=m_{3}=\mu$. Under certain conditions and for very small $\mu$, circular and elliptic keplerian periodic solutions can be continued to ERFBP. For $\mu=1 / 2$, Lyapunov Central theorem can provide a one-parameter family of periodic orbits. Some numerical applications are also shown.

The last one in this category is Nonsphericity of the Moon and Near Sun-Synchronous Polar Lunar Orbits by J. P. S. Carvalho et al.. Here, the dynamics of a lunar artificial satellite perturbed by the nonuniform distribution of mass of the Moon taking into account the oblateness $\left(J_{2}\right)$ and the equatorial ellipticity (sectorial term $C_{22}$ ) is presented. A canonical perturbation method based on Lie-Hori algorithm is used to obtain the second order solutions. A study is performed for the critical inclination and the effect of the coupling terms $\mathrm{J}_{2}$ and $\mathrm{C}_{22}$ are presented. A new second order formula is obtained for the critical inclination as a function of the argument of the pericenter and of the longitude of the ascending node. In the 
same way, for Lunar Sun-synchronous and Near-Polar Orbits, a new formula is obtained to provide the value of the inclination. This formula depends on the semi-major axis, eccentricity and the longitude of the ascending node. For Lunar low altitude satellites, the authors call the attention for the importance of the additional harmonics $\mathrm{J}_{3}, \mathrm{~J}_{5}$, and $\mathrm{C}_{31}$, besides $\mathrm{J}_{2}$ and $\mathrm{C}_{22}$. In particular they mention that, for small inclinations, some contributions of the second order terms can become as large as the first order terms. Several numerical simulations are presented to illustrate the time variation of the eccentricity and inclination.

After that, there are five papers considering the problem of localization with information obtained from space, in particular using GPS and/or GLONASS constellations. The first paper of this topic is GPS Satellites Orbits: Resonance by L. D. D. Ferreira and R. V. Moraes. In this paper, the effects of the perturbations due to resonant geopotential harmonics on the semi major axis of GPS satellites are analyzed. The results show that it is possible to obtain secular perturbations of about $4 \mathrm{~m} /$ day using numerical integration of the Lagrange planetary equations and considering, in the disturbing potential, the main secular resonant coefficients. The paper also shows the amplitudes for the long period terms due to the resonant coefficients for some hypothetical satellites orbiting in the neighborhood of the GPS satellites orbits. The results can be used to perform orbital maneuvers of the GPS satellites to keep them in their nominal orbits.

The second paper is Some Initial Conditions for Disposed Satellites of the Systems GPS and Galileo Constellations by D. M. Sanchez et al.. In this paper the stability of the disposed objects of the GPS and Galileo systems can be affected by the increasing in their eccentricities due to strong resonances. A search for initial conditions where the disposed objects remain at least 250 years, without crossing the orbits of the operational satellites, was performed. As a result, regions where the values of the eccentricity prevent possible risk of collisions have been identified in the phase space. The results also show that the initial inclination of the Moon plays an important role in searching these initial conditions.

Then, we have Quality of TEC Estimated with Mod Ion Using GPS and GLONASS Data by P. O. Camargo. The largest source of error in positioning and navigation with the Global Navigation Satellite System (GNSS) is the ionosphere, which depends on the Total Electron Content (TEC). The quality of the TEC was analyzed taking into account the ModIon model developed in UNESP-Brazil the more appropriate model to be used in the South America region.

After that, we have the paper The Impact on Geographic Location Accuracy due to Different Satellite Orbit Ephemeredes by C. C. Celestino et al.. Here, it is assumed that there are several satellites, hundreds of Data Collection Platforms (DCPs) deployed on ground (fixed or mobile) of a large country (e.g. Brazil), and also some ground reception stations. It considers the question of obtaining the geographic location of these DCPs. In this work, the impact on the geographic location accuracy, when using orbit ephemeris obtained through several sources, is assessed. First, by this evaluation is performed by computer simulation of the Doppler data, corresponding to real existing satellite passes. Then, real Doppler data are used to assess the performance of the location system. The results indicate that the use of precise ephemeris can improve the performance of the calculations involved in this process by reducing the location errors. This conclusion can then be extended to similar location systems.

There is also the paper Simulations under Ideal and Non ideal Conditions for Characterization of a Passive Doppler Geographical Location System Using Extension of Data Reception Network by C. T. Sousa et al.. It presents a Data Reception Network (DRN) software investigation to characterize the passive Doppler Geographical Location (GEOLOC) 
software. The test scenario is composed by Brazilian Data Collection Satellite (SCD2) and the National Oceanic Atmospheric Administration satellite (NOAA-17) passes, a single Data Collecting Platform (DPC) and five ground received stations. The Doppler measurements data of a single satellite pass over a DCP, considering a network of ground reception stations, is the rule of the DNR. The DNR uses an ordering selection method that merges the collected Doppler shift measurements through the stations network in a single file. The pre-processed and analyzed measurement encompasses the DCP signal transmission time and the Doppler shifted signal frequency received on board of the satellite. Thus, the assembly to a single file of the measurements collected, considering a given satellite pass, will contain more information about the full Doppler effect behavior while decreasing the amount of measurement losses, as a consequence, an extended visibility between the relay satellite and the reception stations. The results and analyses were firstly obtained considering the ground stations separately, to characterize their effects in the geographical location result. Six conditions were investigated: ideal simulated conditions, random and bias errors in the Doppler measurements, errors in the satellites ephemeris and errors in the time stamp. To investigate the DNR importance to get more accurate locations, an analysis was performed considering the random errors of $1 \mathrm{HZ}$ in the Doppler measurements. The results show that the developed GEOLOC is operating appropriately under the ideal conditions. The inclusion of biased errors degrades the location results more than the random errors. The random errors are filtered out by the least squares algorithm and they produce mean locations results that tend to zero error, mainly for high sampling rate. The simulations results, considering biased errors, yield errors that degrade the location for high and low sampling rates. The simulation results for ephemeredes error shows that it is fundamental to minimize them, because the location system cannot compensate these errors. The satellites ephemeredes errors are approximately similar in magnitude to their resulting transmitter location errors. The simulations results, using the DRN algorithm, show that to improve the locations results quality it would be necessary to have more Reception Stations spread over the Brazilian territory, to obtain additional amount of data. Then, on the other hand, it improves the geometrical coverage between satellite and DCPs, and better recovers the full Doppler curves, yielding, as a consequence, more valid and improved locations.

A similar problem, but concerned with the determination of an orbit of a satellite, is considered in A Discussion Related to Orbit Determination Using Nonlinear Sigma Point Kalman Filter by P. C. P. M. Pardal et al.. The goal of this work is to present a Kalman filter based on the sigma point unscented transformation, aiming at real-time satellite orbit determination using GPS measurements. Firstly, some underlying material is briefly presented before introducing SPKF (sigma point Kalman filter) and the basic idea of the unscented transformation in which this filter is based. Through the paper, the formulation about orbit determination via GPS, dynamic and observation models and unmodeled acceleration estimation are presented. The SPKF is investigated in many different applications and the results are discussed. The advantages indicate that SPKF can be used as an emerging estimation algorithm to nonlinear system.

Orbital maneuvers for space vehicles are also considered in three papers, as in Orbital Dynamics of a Simple Solar Photon Thruster by A. D. Guerman et al.. This paper studies the orbital dynamics and control for two systems of solar propulsion, a flat solar sail (FSS) and a simple solar photon thruster (SPT). The use of solar pressure to create propulsion can minimize the spacecraft on-board energy consumption during the mission. Modern materials and technologies made this propulsion scheme feasible, and many projects of solar sail are now under development, making the solar sail dynamics the subject of numerous studies. 
To perform the analysis presented in this paper, the equations of the sailcraft's motion are deduced. Comparisons for the performance of two schemes of solar propulsion (Simple Solar Photon Thruster-SSPT and Dual Reflection Solar Photon Thruster-DRSPT) are shown for two test time-optimal control problems of trajectory transfer (Earth-Mars transfer and Earth-Venus transfer). The mathematical model for the force acting on SSPT due to the solar radiation pressure takes into account multiple reflections of the light flux on the sailcraft elements. In this analysis it is assumed that the solar radiation pressure follows inversesquare variation law, the only gravitational field is the one from the Sun (central Newtonian), and the sails are assumed to be ideal reflectors. For a planar motion of an almost flat sail with negligible attitude control errors, the SSPT equations of motion are similar to those for a DRSPT. The analysis showed a better performance of SPT in terms of response time and the results are more pronounced for Earth-Venus transfer. It can be explained by the greater values of the transversal component of the acceleration developed by SSPT compared to FSS.

Then, we have the paper Alternative Transfers to the NEOs 99942 Apophis, 1994 WR12, and 2007 UW1 via Derived Trajectories from Periodic Orbits of Family G by C. F. Melo et al.. This paper explores the existence of a natural and direct link between low Earth orbits and the lunar sphere of the influence to get low-energy transfer trajectory to the three Near Earth Objects through swing-bys with the Moon. The existence of this link is related to a family of retrograde periodic orbits around the Lagrangian equilibrium point $\mathrm{L}_{1}$ predicted by the circular, planar, restricted three-body Earth-Moon-particle problem. Such orbits belong to the so-called Family G. The trajectories in this link are sensitive to small disturbances. This enables them to be conveniently diverted, reducing the cost of a swing-by maneuver. These maneuvers allow a gain in energy enough for the trajectories to escape from the Earth-Moon system and to be stabilized in heliocentric orbits between Earth and Venus or Earth and Mars. The result shows that the required increment of velocity by escape trajectories $\mathrm{G}$ is, in general, fewer than the ones required by conventional transfer (Patched-conic), between $2 \%$ up to $4 \%$. Besides, the spacecraft velocities relative to the asteroids are also, in general, less than that value obtained by the conventional methods. In terms of the transfer time, the results show that in the Apophis and 1994WR12 it is possible to find Closest Point Approaches. The longest time always corresponds to the smallest relative velocity in Closest Point Approaches for trajectories G. Therefore, the trajectories G can intercept the Near Earth Objects orbits and, they can be a good alternative to design future missions destined to the Near Earth Objects.

After that, we have the paper Controlling the Eccentricity of Polar Lunar Orbits with Low-Thrust Propulsion by O. C. Winter et al.. This paper approaches the problem that lunar satellites in polar orbits suffer a high increase on the eccentricity, due to the gravitational perturbation of the Earth leading them to a collision with the Moon. Then, the control of the orbital eccentricity leads to the control of the satellite's lifetime. This paper introduces an approach in order to keep the orbital eccentricity of the satellite at low values. The method presented in the paper considers two systems: the 3-body problem, Moon-Earth-satellite and the 4-body problem, Moon-Earth-Sun-satellite. A system considering a satellite with initial eccentricity equals to 0.0001 and a range of initial altitudes, between $100 \mathrm{~km}$ and $5000 \mathrm{~km}$, is considered. An empirical expression for the length of time needed to occur the collision with the Moon as a function of the initial altitude is derived. The results found for the 3body model were not significantly different from those found for the 4-body model. After that, using low thrust propulsion, it is introduced a correction of the eccentricity every time it reaches the value 0.05 .

Mechanical aspects of spacecrafts are considered in two papers. The first one is Internal Loading Distribution in Statically Loaded Ball Bearings Subjected to an Eccentric Thrust Load 
by M. C. Ricci. In this paper an iterative method is introduced to calculate internal normal ball loads in statically loaded single-row, angular-contact ball bearings, subjected to a known thrust load which is applied to a variable distance from the geometric bearing center line. Numerical examples are shown and compared with the literature. Fifty figures are presented and the results are discussed.

The other paper is The Determination of the Velocities after Impact for the Constrained Bar Problem by A. Fenili et al.. In this paper, a mathematical model for a constrained manipulator is studied. Despite the fact that the model is simple, it has all the important features of the system. A fully plastic impact is considered. Analytical expressions for the velocities of the bodies involved after the collision are derived and used for the numerical integrations of the equations of motion. The theory presented in the paper can be used to problems where the robots have to follow some prescribed patterns or trajectories when in contact with the environment.

One paper deals with the astronomical side of the space dynamics: Gravitational Capture of Asteroids by Gas Drag by E. Vieira-Neto and O. C. Winter. The orbital configuration of the irregular satellites, present in the giant planets system, suggests that these bodies were asteroids in heliocentric orbits that have been captured by the planets. Since this capture is temporary, it has been necessary a dissipative effect in order to turn this temporary capture into a permanent one. This paper deals with this problem by analyzing the effects of the gas drag, from the Solar Nebula, in the orbital evolution of these asteroids after they have being captured by the planets. The results show that, although this dissipative effect is important, it is not the only mechanism responsible for keeping the asteroids in a permanent orbit about the planet.

Then, we also have one paper studying the motion of a spacecraft when traveling in the atmospheric region of the space: Atmospheric Reentry Dynamics of Conic Objects by J. P. Saldia et al.. In this paper, the accurate determination of the aerodynamics coefficients is an important issue in the calculation of the reentry trajectories of an object inside the terrestrial atmosphere. The methodology to calculate these coefficients and how to include them in a code, in order to compute the reentry trajectories, is considered. As a result, a sample of trajectories of conical objects for different initial flight conditions is presented.

\section{Acknowledgments}

The guest editors would like to thank all the authors, the reviews, the Editor of the journal, and all the staff involved in the preparation of this issue for the opportunity to publish the articles related to this important subject. 


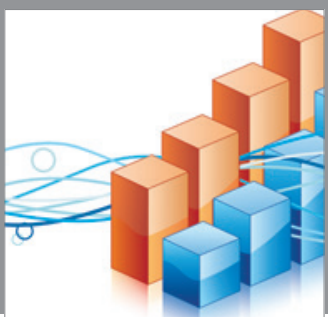

Advances in

Operations Research

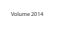

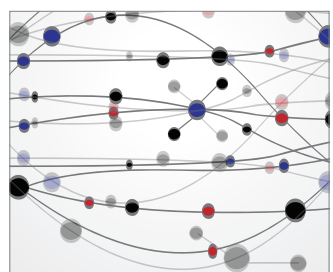

\section{The Scientific} World Journal
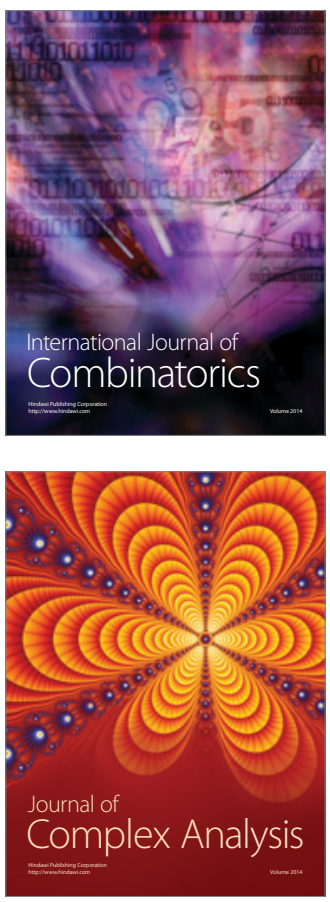

International Journal of

Mathematics and

Mathematical

Sciences
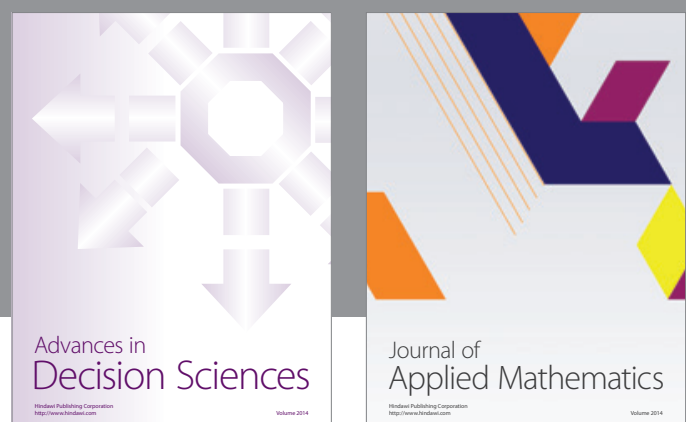

Journal of

Applied Mathematics
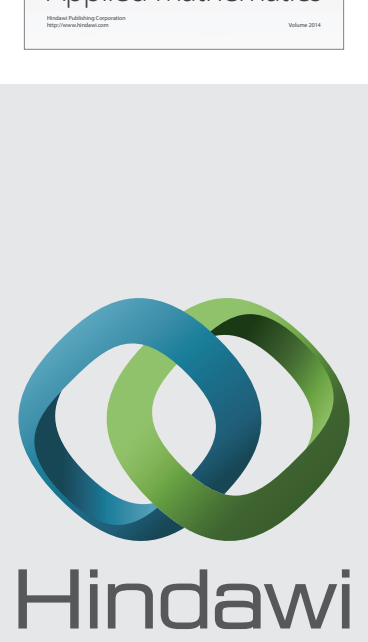

Submit your manuscripts at http://www.hindawi.com
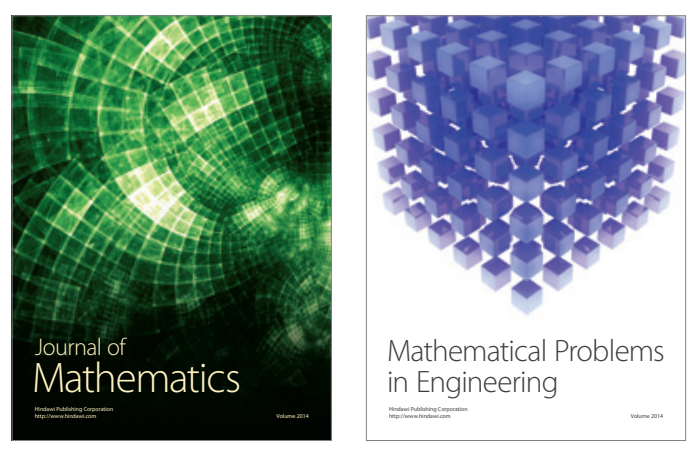

Mathematical Problems in Engineering
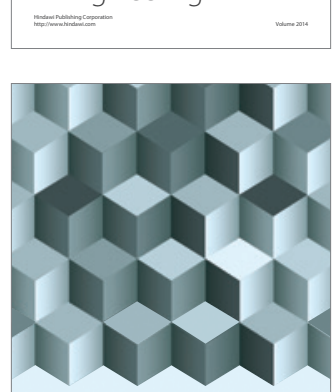

Journal of

Function Spaces
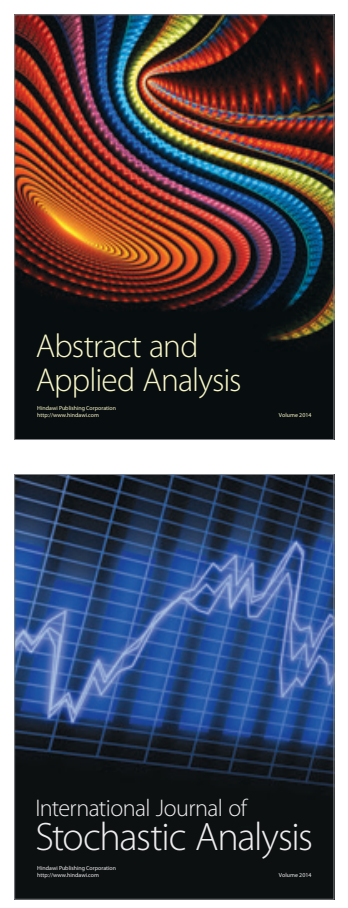

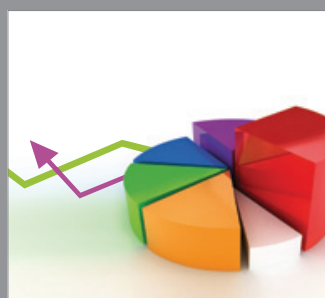

ournal of

Probability and Statistics

Promensencen
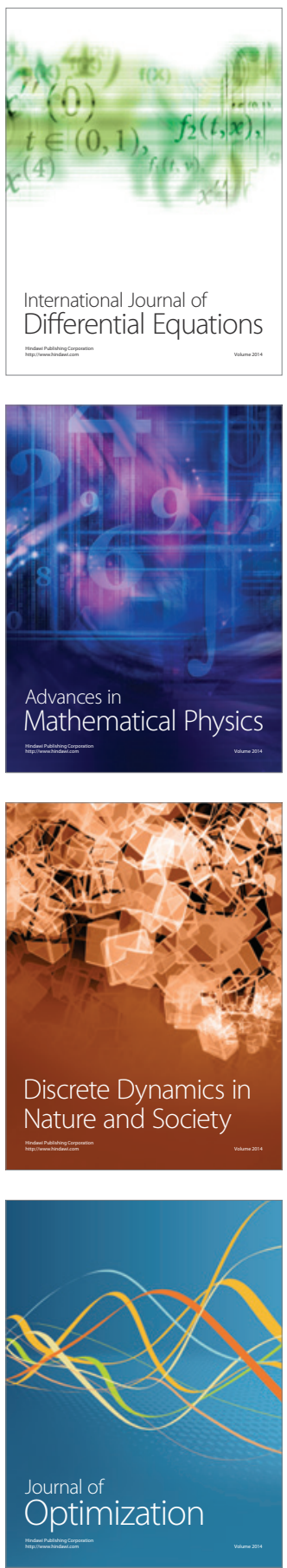